\title{
¿QUIEN QUIERE GENERO CUANDO PUEDE TENER SEXO?
}

\author{
LUCY GARRIDO
}

\author{
Cotidiano Mujer
}

\begin{abstract}
Resumen: A partir de un paneo sobre la definición de red y del trabajo en redes del movimiento feminista, y de algunas de las últimas experiencias de coordinación regional, el artículo plantea la interrogante "Articular cómo, desde dónde y para qué". Se sugieren tres grandes líneas de trabajo que en varios casos guían o podrían guiar la acción feminista regional. La inserción en el Foro Social Mundial forma parte de la estrategia de incidencia que la Articulación Feminista Marcosur se ha dado para contestar esa pregunta. Y la elección del tema y el estilo de la campaña "Tu boca fundamental contra los fundamentalismos", es una manera de responder a la del título: "quién quiere género cuando puede tener sexo?"
\end{abstract}

Palabras claves: movimiento feminista, estrategias, articulación, comunicación.

Cuando supe del tema del que me había tocado hablar ("Redes y líneas de trabajo") y, para peor, escribir quince páginas (a mí, que hablo y escribo en pocas palabras), estuve a punto de no venir. ' Cecilia Olea me hizo pensar que a lo mejor se trataba de "redes de pesca artesanal" $y$, como pescar me encanta, empecé a ver la tarea con más cariño. Claro que con lo de "líneas de trabajo" se complicaba un poco (pescar me gusta, trabajar no). Pero bueno, al menos nadie había pedido que escribiera sobre "los nudos del feminismo" a alguien que, a todas luces, prefiere los "desnudos".

En el Diccionario de la Real Academia Española, la palabra RED tiene varias acepciones: "Aparejo hecho con hilos, cuerdas o alambres trabados en forma de mallas, y convenientemente dispuesto para pescar, cazar, cercar, sujetar", es uno de ellos. Y aunque parezca que no tiene nada que ver con las redes de los movimientos sociales, estos verbos son dignos de atención: alguna vez al menos, hemos sabido de redes que "sujetaban" a sus miembros para "pescar" financiamientos.

Deseché la acepción que dice que red es "un ardid o engaño de que uno se vale para atraer a otro" porque entendí que la Real Academia se estaba refiriendo a las arañas (?) o a la red que podemos tender para enamorar a alguien (?), pero de ninguna manera a las feministas. Y también deseché la que el diccionario define como un "conjunto de personas relacionadas para un fin común, por lo general de carácter secreto, ilegal o delictivo", por razones obvias: si alguna red estuviera haciendo algo así, las agencias ya no seguirían preguntando cuáles son los planes que tenemos para el autofinanciamiento.

Copyright (c) 2003 by Revista Estudos Feministas

' Ponencia para el "Seminario Feminismos latinoamericanos: retos y perspectivas", organizado por el PUEG en México, del 22 al 26 de a bril del 2002. Fue publicada en Debate Feminista, n. 27, abril, 2003. 
Entonces me quedé con esta definición de red: "Conjunto y trabazón de cosas que obran a favor o en contra de un fin o de un intento". Y ahí sí reconocí ese estilo de organización que gran parte del movimiento se ha dado.

A partir del I Encuentro Feminista de América Latina y el Caribe, las feministas comenzamos a "enredarnos" de una manera informal: verse las caras, conocerse, discutir, posibilitó el intercambio de experiencias y estrategias y abrió el campo de la coordinación y articulación de acciones. Hasta el día de hoy, todas las feministas de la región (aquéllas con estas, aquéllas otras con las de más allá que se juntan con las de acá y se separan de las que tal y cual) estamos "enredadas" en el sentido de ser uno y varios conjuntos que obramos a favor o en contra de un fin. Y también en el sentido de "enredo", que la Real Academia define como "complicación y maraña que resulta de trabarse entre sí desordenadamente...". Pero este aspecto mejor no lo tocamos, al menos, por ahora.

La creación de las redes "formales" en la región, es decir, las que se autodefinen como redes, tienen estatutos, estructura y vocación de coordinar a sus miembros, comienza en 1984 con la Red de Salud de las Mujeres Latinoamericanas y del Caribe. En 1988 se crea la REPEM (Red de Educación Popular Entre Mujeres); luego, la Red Latinoamericana y del Caribe Contra la Violencia Doméstica y Sexual, el CLADEM (Comité de América Latina y el Caribe para la Defensa de los Derechos de la Mujer) y varias otras redes temáticas. Algo después nacen las redes llamadas de "identidad" y de "sectores específicos": las de las mujeres indígenas, afrodescendientes, parlamentarias, comunicadoras, municipales, etc. Cada red con su agenda y el objetivo de darle a esa agenda una presencia regional e internacional que, a su vez, fortaleciera a sus integrantes a nivel nacional, intercambiando información, conocimientos y recursos. Nada de eso puede hacerse sin planes comunes, sin confianza y, claro está, sin estructura y "cierto grado" de institucionalización.

Las redes lograron visibilizar demandas feministas, han aportado conocimiento, diagnósticos y propuestas, han sido actoras importantísimas en la articulación de los movimientos de mujeres, en el reconocimiento de la diversidad y han contribuido a cuestionar la versión de que éramos todas iguales y el feminismo uno solo.

Pero también el camino recorrido tiene sus tropiezos. Uno de ellos es que ese "cierto grado" de institucionalización se hizo a veces difícil de controlar. Eso llevó a que en algunas redes se privilegiara la estructura y la sede; que en vez de administrar contenidos y recursos, los centralizaran, alejándose cada vez más de la primigenia intención de horizontalidad. Otro tropiezo es que trabajar en redes temáticas o de identidad puede hacerse atávico y corporativo. Entonces las que están en el tema de la salud reproductiva a veces "no ven" que la feminización de la pobreza va más allá de la planificación familiar; o las que están en la red de indígenas "no se enteran" que en ese momento las lesbianas están llevando adelante tal o cual acción a las que les convendría estar atentas, y así sucesivamente. Por otra parte, cuando las membrecías no están claras, la "virtualidad" deja de ser monopolio de las redes del ciberespacio.

En 1994 gran parte del movimiento feminista decide participar de la IV Conferencia de Naciones Unidas sobre la Mujer. Pero el Foro de Mar del Plata, junto al entusiasmo y el esfuerzo por llegar de un modo organizado a Beijing, también hizo "evidentes" errores y horrores. Pese a eso, las discusiones que mantuvimos y las decisiones que tomamos en él hicieron que llegáramos a la Conferencia con una coordinación regional que fue la mayor experiencia de articulación feminista que hayamos tenido hasta ahora. ${ }^{2}$ En el proceso hacia Beijing se fueron creando espacios de confluencia que facilitaron un mayor intercambio entre las redes temáticas, sectoriales y de identidad, pero además surgió una

2 Line BAREIRO, 2000. 
nueva manera de organizarnos: las coordinaciones nacionales y subregionales posibilitaron una agenda regional que iba más allá de la suma de todas las agendas y habilitaron una postura común, un marco político y una estrategia capaz de influir en la agenda global.

Por supuesto, no creo que hayamos logrado crear una red donde el centro "estuviera en todas partes". Pero fue una articulación lo suficientemente flexible como para que, con excepciones, con diferencias de una subregión a otra, con tropiezos y encontronazos, pudiéramos trabajar de la manera más democrática, participativa y horizontal que se pudo. Fue un proceso en el que las diversidades y las diferencias, sorteando el riesgo de "la pulverización del sujeto del movimiento feminista", ${ }^{3}$ se administraron de tal manera que nos enriquecieron e hicieron ganar fuerza. Sin embargo, cuando en 1997 se creó la Articulación de ONGs de América Latina y el Caribe, no se pudo sostener la estrategia del "pretexto" que nos habíamos fijado en el proceso hacia la Conferencia y ni los objetivos, ni las formas organizativas que se dieron antes, pudieron ir más allá de organizar la presencia en CEPAL 2000 y en Beijing +5 .

En la conferencia de Nueva York que evaluaba los avances y retrocesos de Beijing, también nosotras como feministas tuvimos la oportunidad de evaluar hasta qué punto éramos parte de un movimiento con conciencia de ser "regional". Porque no sólo las redes del norte son más fuertes sino que hay redes globales que tienen más recursos y más influencia, más poder para decidir temas, rumbos y estrategias. Y lo que sus expertas deciden para ellas, debe ser bueno para todas; y en el momento en que los gobiernos se hacían los sordos y retrasaban la aprobación del documento, ellas habían decidido que todo el mundo (incluidas nosotras) debía mantener la estrategia del advocacy y el lobby y, por tanto, que en el discurso que las ONGs de América Latina y el Caribe iban a dar en la Asamblea General, teníamos que hablar de tales y cuales temas y con tal y cual tono.

Pero tantas letras tiene un "sí" como un "no". Y nosotras no quisimos más advocacy, quisimos movimiento. Hicimos y dijimos lo que se nos dio la gana: "Señoras y señores delegados.... ¿Cómo, después de esta patética inoperancia, volver a creer en aquéllos gobiernos cuyas elites políticas responden solamente a sus intereses religiosos, políticos y económicos, particulares, pequeños y mezquinos?"

En estos tiempos de globalización, tensiones como esa vamos a seguir viviéndolas. Pero tenemos que estar atentas: una cosa es globalizar las luchas feministas y otra es que seamos "globalizadas" por otras feministas.

(No deja de ser interesante que tanto en el Encuentro Feminista de Cartagena como en la Conferencia de Nueva York, unas nos acusaran de no ser autónomas y las otras de serlo en exceso. Para defender su punto de vista, algunas mujeres en Chile eligieron el insulto y el grito; otras, en Nueva York, intentaron desconocer y silenciar a las que pensábamos diferente.)

\section{¿Pero articular cómo, desde dónde y para qué?}

Cuando en el seminario "Mujer y democracia en el MERCOSUR" (Montevídeo, setiembre 2000) se discute la necesidad de consolidar y potenciar las experiencias, el conocimiento y la confianza acumulados en el proceso a Beijing, nace la Articulación Feminista MARCOSUR con una agenda abierta, que tiene la posibilidad de recrearse a partir de la discusión de acciones prioritarias y sin ahogar los intereses específicos de sus integrantes. La AFM se define como una "corriente de pensamiento y acción feminista que

${ }^{3}$ Celia , 1999. 
quiere incidir políticamente en las relaciones de cultura y poder presentes en los procesos de integración regional y en los grandes debates internacionales". ${ }^{4}$

Podría haberse llamado Articulación Feminista "MERCOSUR" por el interés que tenemos en los temas de la región y porque están integradas coordinaciones nacionales de los países que lo conforman. Pero se llama MARCOSUR porque la integración que nos interesa va más allá del comercio y los mercados y porque se trata de un "marco maestro", en el sentido al que se refieren Snow y Benford. ${ }^{5}$ Es decir, tenemos una forma de interpretación basada en una serie de valores compartidos, que van más allá de un contexto específico y de cualquier frontera. Por eso también la integran grupos de Perú y Bolivia, redes regionales o feministas a título personal. Es un espacio, un proceso que a medida que avanza, se perfila; no tenemos problema ninguno en decir que a veces más que a una "corriente de pensamiento" nos parecemos a una "síntesis de intuiciones".

Según Andrés Servin, ${ }^{6}$ la principal diferencia entre los viejos y los nuevos movimientos sociales es que los primeros se organizaban en torno a divisiones de carácter clasista (movimiento obrero, movimiento campesino) y los de ahora en torno a contradicciones sociales como el género, el medioambiente, las etnias, los conflictos bélicos, etc. Aunque de todas maneras habría que ver si el movimiento feminista y de mujeres no tiene un sujeto histórico determinado estructuralmente, el hecho es que estos nuevos movimientos (articulados en redes regionales y transnacionales, en torno a intereses globales pero con raíces e intereses locales) dan origen a la llamada "sociedad civil global", que a su vez" reproduce en sus interacciones los conflictos y contradicciones de las sociedades civiles domésticas. El mejor ejemplo de todo esto es el Foro Social Mundial.

Participar como Articulación Feminista MARCOSUR en la construcción del FSM desde su inicio, insertando en el debate nuestros temas, siendo contestatarias y creativas y aportando nuestro aprendizaje en torno a la construcción política de agendas, es una manera de contestar a esa pregunta de "articular cómo, desde dónde y para qué".

El FSM expresa "inevitablemente las tensiones internas y externas de los movimientos sociales globales, es un hábitat que revela e incluso reproduce rasgos y prácticas ambiguas, cruzadas por movimientos democratizadores y bolsones de autoritarismo, sexismo, o racismo". Y allí mismo, por pura intuición, fuimos a insertarnos.

Porque es un espacio donde confluyen las protestas con las esperanzas y el desasosiego con la construcción de alternativas. Donde están los del Movimiento Sin Tierra y los que viven sin techo y sin ventana, los movimientos indígenas y afrodescendientes junto a los jóvenes y los economistas, los transexuales y las feministas. Los que dan masajes holísticos, con los académicos. Los que luchan contra los productos transgénicos y los que son transgénero. Hindúes, musulmanes, judíos, católicos junto a los sindicalistas y la gente que promueve el esperanto como lenguaje universal.

Es un cambalache. Es un enorme caos. Pero es un caos muy creativo que está buscando responder a esta globalización no con una receta dada por un solo sujeto, sino haciendo confluir las búsquedas desde un espacio descentralizado, diverso y plural. Y por esas mismas razones es que también en él confluyen viejas y nuevas formas del pensamiento, modernas y anquilosadas maneras de actuar en un proceso colectivo que se construye con movimientos, redes, campañas, alianzas.

\footnotetext{
${ }^{4}$ DOCUMENTO DE LA ARTICULACIÓN..., 2001.

${ }^{5}$ Citados en Kate NASH, 2000, p. 124-125.

${ }^{6}$ SERVIN, 1977.

7 Virginia VARGAS, 2002.
} 
"Estamos frente a la necesidad de radicalizar la perspectiva de los derechos humanos DE TODOS LOS HUMANOS como prioridad fundamental para dar cuenta de una nueva conciencia de la humanidad. Romper el divorcio entre economía y sociedad, entre economía y naturaleza, entre naturaleza y sociedad son tareas centrales para la construcción de una agenda global promotora de una ciudadanía planetaria", dice Cándido Grzybowski. ${ }^{8}$

Como Articulación Feminista MARCOSUR privilegiamos el FSM porque es un espacio político en por lo menos tres sentidos que nos son fundamentales: uno de ellos es que queremos que la agenda feminista (la subversión simbólico-cultural, los derechos sexuales, la equidad, etc.) forme realmente parte de la agenda por la justicia económica y la profundización de la democracia; otro sentido es que para lograrlo debemos dar la batalla al interior mismo del Foro, disputando contenidos y siendo subversivas también en él; y, por último, porque el Foro es amplificador de nuestros propios discursos como lo probamos con la campaña "Tu boca, fundamental contra los fundamentalismos" que, en Porto Alegre, fue acompañada activamente por otros movimientos y redes de mujeres, por los jóvenes, los sindicalistas, etc. Y criticada también por los que decían que le estábamos haciendo el juego a Bush, que las bocas eran muy eróticas y, por lo tanto, estábamos usando un símbolo demasiado light (¿el erotismo es light? ¿desde cuándo?) o que materiales de esa calidad debía estar financiándolos la CIA.

Pero para trabajar en la construcción de una alianza con otros movimientos no sólo es imprescindible que la coyuntura indique la oportunidad de hacerlo, se necesita también de "la presencia de esquemas de interpretación y significados compartidos" con esos otros actores y de "marcos de acción colectiva" con los que se pueda promover la movilización. Antes aún, se necesita sortear el tema de la legitimidad y el reconocimiento de cada actor, no sólo ante su propia base social sino ante los otros. Y esto nos lleva al tema de las líneas de trabajo y de la construcción de nuestra agenda.

Una agenda debe tener principios fundantes. No puede ser un listado de todos los intereses según seamos negras, blancas, heterosexuales, académicas, lesbianas o de Piscis sino que debe ser una especie de "sentido común", de acuerdos básicos sobre las metas y las estrategias que nos ayudarán a conseguirlos. ¿Cuál es ese principio fundante y fundamental? Que "lo personal es político" y de él es que luego se derivan los "temas sustantivos" como: a) los que se refieren a la pobreza, la desocupación, etc.; b) los que tienen que ver con los derechos sexuales y los reproductivos; c) la vinculación de la violencia contra las mujeres como un tema de derechos humanos; d) todo lo que tiene que ver con la no discriminación, la igualdad, etc.

Y hay otro grupo de temas más "posicionales o procedimentales"9 (porque tienen que ver con el lugar de las mujeres y no necesariamente con los temas en sí mismos). En estos temas se ubican, por un lado, los que se relacionan con las demandas por una mayor participación (por ejemplo, una ley de cupos electorales) y, por otro, con la inserción de nuestros temas en los distintos movimientos y en la agenda pública.

Hay por lo menos cuatro actores que construyen agendas y que a la vez son factores determinantes para la construcción de las agendas de los otros: el sistema político, los medios de comunicación, el campo internacional y, el más débil de todos, el campo ciudadano, donde están los movimientos sociales.

¿Cómo nos articulamos las mujeres, las feministas, con los otros movimientos? ¿Cómo articulamos nuestra agenda con la de ellos? ¿Cuántos feminismos somos? ¿Cuántas agendas

\footnotetext{
${ }^{8}$ Director de la ONG brasilera IBASE, integrante del Comité Organizador del Foro Social Mundial.

${ }^{9}$ Constanza
} 
tenemos? Hemos pasado de una primera etapa homogénea y un poco naï, la verdad sea dicha, a una heterogeneidad que será todo lo enriquecedora que se quiera (aquello de lo que Gina Vargas habla tan poéticamente como "los múltiples espacios y las múltiples estrategias"), pero que genera diversidades y diferencias que dificultan mucho la articulación entre nosotras mismas.

Sin embargo, no hay que desesperarse: eso también le pasa a otros movimientos (preguntémosle al sindical qué fue de la homogeneidad de la clase obrera) Sólo que nosotras, las reinas de la diversidad, las reinas de las diferencias, aún no sabemos muy bien cómo administrarlas y llevamos el pluralismo y la necesidad de "estar en todas" al nivel de la esquizofrenia.

En un artículo ${ }^{10}$ decía que el que hubiéramos sabido movernos en el proceso hacia Beijing, ubicando nuevos escenarios y actores, tomando de lo global aquello que servía para organizarnos más como región y fortaleciendo las demandas y propuestas que hacíamos en nuestros países, había sido un acierto y lo sostengo. Pero el título de ese artículo era "Bye bye Beijing" y no sólo porque me divirtiera el juego de palabras.

Siempre es imprescindible que muchas organizaciones trabajen volcadas hacia las políticas públicas, que se conquisten mejores leyes, reformas y avances concretos, que haya más diputadas. Siempre va a ser fundamental que logremos mayor reconocimiento de los estados y que en el debate sobre democracia insistamos con firmeza en incorporar la equidad y el género. Pero en estos años también pudimos constatar que algunos temas son más cómodos de luchar que otros y que es más fácil encontrar aliados contra la violencia doméstica y sexual que a favor del derecho al placer, la opción/orientación sexual o la legalización del aborto.

Pues bien, estamos en el 2002 y somos feministas. ¿Por qué, parodiando a Goetz, no damos vuelta su provocación y desde el movimiento nos preguntamos QUIEN QUIERE GENERO CUANDO PUEDE TENER SEXO? ${ }^{11}$ Decía en ese artículo que hay palabras que simbolizan procesos y se ponen en boga. A "empoderamiento" le siguió "lobby", a "accountability", "monitorear", y me preguntaba cuáles serán las palabras que sintetizarán nuestros discursos y nuestras acciones, nuestra agenda en esta nueva etapa.

Creo que deberían ser lo suficientemente provocadoras como para que revitalicemos el carácter subversivo que hace 20 años fue más fácil tener. Dicho esto sin caer en la dicotomía simplista "revolución o reforma", dicho sin ninguna nostalgia, dicho desde el conocimiento y la fuerza que acumulamos en esta década.

El objetivo fue y sigue siendo el cambio cultural y entonces no tengo la menor duda de que la palabra "sexo" tiene que ser una de ellas.

\section{En esta agenda, que no será la agenda de todas sino apenas de todas las que se pongan de acuerdo en ella, hay por lo menos tres grandes líneas de trabajo, cada una con su grado de dificultad a la hora de articularla con otros sectores.}

1. La pobreza y la desigualdad, la justicia, están en esa agenda y el marco de los derechos económicos, sociales y culturales con cierta facilidad nos permite participar de esa otra "agenda común" con otros movimientos. El factor que favorece más esta inclusión transversal es, obviamente, el viejo y querido "enemigo común". El modelo económico

10 LucY GARRIDO, 2000.

1 Sally BADEN y Anne Marie GOETZ, 1997. 
contra el que todos estamos, hace que la injusticia y la desigualdad sean temas de discusión y debate, aunque, paradójicamente, es ese mismo modelo el que no sólo fragmenta el mundo del trabajo sino también el comunitario y el mundo cultural. Por eso tenemos que insistir en tomar a los DESC no desde su lado técnico - que lo haremos -, no desde el punto de vista legal - que también lo haremos -, sino fundamentalmente como un desafío político para acercarlos a la realidad, a la vida diaria de todas las mujeres y de todos los hombres.

Hay que hacer de los DESC "derechos globales". Es decir, "¿querías sopa? Acá tenés dos platos". ¿La OMC, el ALCA, los "mercaderes del templo", querían globalización? Hay que darles dos platos. Y ya se están llevando adelante varias iniciativas en este sentido. Muchas feministas están produciendo conocimiento y movilización respecto a los ajustes estructurales y el modelo de desarrollo, como la coalición contra el ALCA o la Iniciativa Feminista de Cartagena; es de esperar también que el proceso para la Conferencia de Durban haya dejado puentes tendidos para plataformas y estrategias comunes contra todo tipo de discriminación.

2. Hemos logrado desprivatizar la violación de los derechos humanos dentro del hogar y, en ese marco, se están abriendo mejores posibilidades de trabajo conjunto de las que había antes y varios empiezan "a introducir una visión género-sensitiva en su accionar", al decir de Susana Chiarotti. Iniciativas que están llevando adelante redes como CLADEM, grupos como La Morada o el Instituto de la Mujer en Chile o los que integran Causa Abierta, todos denunciando a los estados ante la Corte Interamericana de Derechos Humanos por incumplimiento de la Convención de Belem do Pará o, incluso, como cómplices de la violencia contra las mujeres. Están colaborando, además, en ampliar el cause para que otros movimientos (indígenas, afrodescendientes, homosexuales) también demanden a los estados.

Después de tantos años, lo que dijo Eleanor Roosevelt sigue teniendo sentido: "¿Dónde, después de todo, comienzan los derechos humanos universales? En los pequeños lugares, cercanos al hogar, tan cercanos y tan pequeños que no pueden verse en ningún mapa del mundo. $Y$ sin embargo, son el mundo de la persona individual: el barrio en que se vive, el colegio o la universidad a los que se asiste, la fábrica, las granjas o la oficina en donde se trabaja. Estos son los lugares en donde todo hombre, mujer o niño busca igualdad ante la justicia, igualdad de oportunidades, igualdad de dignidad, sin discriminación. A menos que estos derechos cobren sentido allí, tendrán muy poco sentido en cualquier otra parte."12

3. Desde los derechos sexuales y los derechos reproductivos tal vez sea un poco más complicada la elaboración de alianzas. Requiere de posturas amplias, plurales, flexibles, características todavía difíciles de encontrar entre muchos dirigentes de los movimientos sociales, incluyendo el de las mujeres y el feminista. Todo depende, como siempre, del proceso que seamos capaces de hacer en la región: una campaña por una convención interamericana de los derechos sexuales y los derechos reproductivos obligaría a las personas, a las organizaciones y los movimientos sociales (empezando por el nuestro) a pronunciarse sobre temas que aún les producen "contradicciones", obligaría a reconocer los desacuerdos y a comprometerse con los acuerdos. Y desde el punto de vista estratégico, organiza en un camino común, esfuerzos que a veces aparecen desperdigados perdiendo efectividad.

La Campaña por la Convención está obstinada en dar ese debate y en generar movimiento, en que la opción sexual no forme parte de un listado temático que luego podremos olvidar, en que los derechos sexuales no queden subsumidos en los reproductivos

\footnotetext{
${ }^{12}$ Eleanor , 1958.
} 
en un movimiento en el que, ioh paradoja! , las mujeres aman a otras mujeres con bastante naturalidad... Una campaña sobre este tipo de derechos puede introducir, en la mitad de la agenda común y global, un modo "subversivo" de apostar a los cambios culturales, esos que sólo pueden darse cuando nos proponemos cambiar la cabeza de la gente.

"La ciudadanía - dice Betânia Ávila13 - debe ser tomada como un valor que motive la revuelta, oriente el camino y ofrezca los medios de mejorar la vida que está siendo vivida. Debe servir como inspiración para tomar parte en el conflicto de la transformación social, para producir el discurso de los sujetos y para no aceptar más la violencia de sexo, de raza o de clase, como parte de un destino".

Desde estas tres grandes líneas de trabajo y partiendo de lo que dice Betânia, ¿cuáles podrían ser los mensajes? ¿en qué tono hablaremos? Ante tanto fundamentalista de todo tipo que anda suelto por ahí, ino es hora de hacerlo desde la contra-cultura, desde la movilización, desde la subversión?.

Sé que puede parecer contradictorio que, por un lado, defienda la contra-cultura y, por otro, hable de trabajar hacia una "convención" que a todas luces, debe ser lo más aburrido que hay. iPero es que creo que eso debemos hacerlo de la manera más anticonvencional del mundo!.

Por ejemplo, cada vez que hablamos del aborto o de los derechos sexuales, "valerosa y estoicamente" toleramos que la lglesia, cuyo territorio es "el reino de los cielos", dé cátedra sobre los temas terrenales. ¿Por qué no dar vuelta a las cosas y meternos nosotras en el área de ellos? ¿Qué llama más la atención de la gente y de los medios? ¿Que las feministas hagamos la mesa redonda número 3.760 para hablar de la violencia sexual o que organicemos una sobre "Violación de menores en la Santa Madre Iglesia" o sobre "El sujeto omitido en la Santísima Trinidad"? ${ }^{14}$ ¿Y por qué no una charla pública sobre "Placeres y Demonios"? Se pondría muy interesante si empezáramos por decir que, gracias al Antiguo Testamento, es irrefutable la superioridad de Eva sobre Adán, puesto que éste nace del barro y aquélla de materia orgánica. Y que tan superior es que para que Adán pecara fue suficiente tentación la existencia de la mujer, en cambio, para que Eva se decidiera tuvo que venir el mismo demonio a convencerla. Con el pobre Adán, el demonio ni siquiera habló. Sin embargo, con la cantidad de curas y obispos que están siendo denunciados ahora por violar menores, parece que el demonio tiene una línea dedicada, una comunicación muy fluida...

Si es que queremos tejer alianzas con otros movimientos, tenemos que hacerlo desde una agenda propia, que cuestione, provoque y desafíe el debate público "colocando en el centro de la profundización democrática la libertad de las personas, su corporalidad y su sexualidad, como el derecho básico a tener derechos". ${ }^{15}$

Porque sigue siendo cierto: no hay nada más político que lo personal y porque abrir las cabezas y los corazones de la gente es un eje de resistencia enorme contra los defensores del pensamiento único.

Pero un discurso así, sólo podemos hacerlo si estamos dispuestas a producir nuevos

\footnotetext{
${ }^{13}$ Maria Betânia ÁVILA, 2001, p. 59.

${ }^{14}$ El Padre, el Hijo y el Espíritu Santo... ¿No falta algo?

15 Lilián CELIBERTI, 2001.
} 
símbolos que fortalezcan las utopías, a construir futuro sabiendo que la vida es hoy, que la exclusión, la pobreza y la desigualdad son hoy. Que los sueños, el deseo y la felicidad, también son hoy. Y es hoy que el discurso de los derechos debe, de una buena vez, salir de la abstracción a la calle, a cada casa, a cada cama, a cada persona.

\section{Referencias bibliográficas}

AMORÓS, Celia. "La idea de igualdad". Especial Fempress, 1999. p. 11-12.

ÁVILA, Maria Betânia. "Feminismo, cidadania e transformação social". In: Textos e

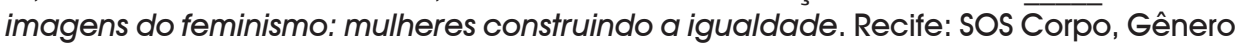
e Cidadania, 2001.

BADEN, Sally, and GOETZ, Anne Marie. "Who Needs [Sex] When You Can Have [Gender]? Conflicting Discourses on Gender at Beijing." In: STAUDT, Kathleen (ed.). Women, International Development, and Politics: The Bureaucratic Mire. Philadelphia: Temple University Press, 1997. p. 37-58.

BAREIRO, Line. "Cambios para el conjunto de la sociedad". In: GOMÁRIZ, Enrique; MEENTZEN, Angela (Eds.). Democracia de género: una propuesta para mujeres y hombres del siglo XXI. San José: Fundación Género y Sociedad (GESO) y Fundación Heinrich Boell (FHB), 2000. p. 77-81.

CELIBERTI, Lilián. "Los gozos y las sombras". Jornal da Rede Feminista de Saúde, diciembre 2001. Documento de la web: <http://www.redesaude.org.br/jr24/html/jr24-lilian.html> .

DOCUMENTO DE LA ARTICULACIÓN FEMINISTA MARCOSUR. Septiembre, 2001.

GARRIDO, Lucy. "Bye bye Beijing". Cotidiano Mujer, mayo 2000. Documento de la web: $<$ www.cotidianomujer.org.uy/2000/32_p3.htm>.

NASH, Kate. Contemporary Political Sociology: Globalization, Politics and Power. Oxford: Blackwell, 2000.

MOREIRA, Constanza. Comentarios a la investigación de Niki Jonson "Agenda de género y articulación en los movimientos sociales uruguayos ante los desafíos del siglo XXI", para Cotidiano Mujer y el Programa Mujer y Democracia en el Cono Sur. Documento de la web: <www.cotidianomujer.org.uy $>$.

ROOSEVELT, Eleanor. En la presentación de "In your Hands: A Guide for Community Action for the Tenth Anniversary of the Universal Declaration of Human Rights." United Nations, New York. March 27, 1958. Documento de la web: <www.udhr.org/history/inyour.htm.

SERBIN, Andrés. "Globalización y sociedad civil en los procesos de integración". Nueva Sociedade, n. 147, p. 44-55, enero-febrero 1997.

VARGAS, Virginia. "El II Foro Social Mundial". La República de las Mujeres, Uruguay, febrero 2002. Documento de la web: http://www.convencion.org.uy/08Debates/ DocumentosWord/EIFSGina.doc.

\footnotetext{
Who Wants Gender When You Can Have Sex?

Abstract: Based on an overview about the definition of both network and work within feminist movement networks, and about some of the last regional coordination experiences, the article sets out the question: "Articulation: how, where from and what for". Three large working guidelines are suggested, that, in several cases, guide or might guide regional feminist action. Insertion in World Social Forum takes part of the incidence strategy that Marcosur Feminist Articulation has adopted in order to answer this question. And election of subject matter and style of the campaign "Your mouth, fundamental against fundamentalisms", is a way to respond to the one that says: "who wants gender, when one can have sex?"
}

Key words: feminist movement, strategy, articulation, communication. 